\title{
Die Gutachterausbildung in der Schweiz: aktuelle Standortbestimmung und Ausblick
}

\section{Hans Rudolf Stöcklia , Roger Darioli ${ }^{b}$}

a Dr. med., Leiter Gutachterkurse

b Prof. Dr. med., Leiter Gutachterkurse Romandie
1 Meine J. Ärztliche Begutachtung in der Schweiz - Erfüllt sie die heutigen Qualitätsanforderungen? Swiss Surgery. 1998;4:53-7.

2 Ludwig CA. Qualität fachärztlicher Gutachten im Bereich der sozialen Unfallversicherung. Medizinische Mitteilung. SUVA Nr. 77/2006;5-15.

3 Stöckli H.R. Die Ausbildung medizinischer Gutachter in der Schweiz - bisher und zukünftig. Schweiz Ärztezeitung. 2006;87(23):1030-3.

\section{Korrespondenz:}

Dr. med. Hans Rudolf Stöckli

Facharzt für Neurologie FMH

Leiter Gutachterkurse

Neurologe Kantonsspital

und Kantonale Psychiatrische Klinik

Liestal

Neurologische Konsiliararztpraxis

Liestal

Kasernenstrasse 22a

CH-4410 Liestal

hrstoeckli@datacomm.ch
Die Anforderungen an medizinische Gutachter sind in den letzten Jahren stetig gestiegen, der Bedarf an gut ausgebildeten medizinischen Gutachtern hat dauernd zugenommen, gleichzeitig haben zwei Studien gezeigt, dass die Gutachtenqualität sehr zu wünschen übrig lässt. So wurde 1998 von 262 Gutachten aus dem schweizerischen Privatversicherungsbereich etwa je ein Drittel als einwandfrei, lückenhaft oder schwer mangelhaft taxiert [1]. 2006 kam eine SUVAinterne Evaluationsstudie von 102 externen Gutachten zu ähnlichen Resultaten: $5 \%$ perfekt, $46 \%$ tauglich, 19\% mangelhaft, 30\% untauglich [2]. Die Schaffung einer zentral geleiteten SUVA-internen Gutachtenevaluationsstelle war die Folge.

Die Problematik des Gutachterwesens erkennend, hat die FMH 2005 die Swiss Insurance Medicine (SIM) beauftragt, die von der SUVA und vom Schweizerischen Versicherungsverband 1998 aufgenommenen Gutachterkurse, die sich lediglich auf den UV-Bereich bezogen, auf alle Versicherungszweige auszudehnen (UV, IV, MV, $\mathrm{KV}$, Privatversicherung, Haftpflicht) und die zukünftigen Ausbildungskurse unter Einbezug aller am Gutachterwesen Beteiligten neutral durchzuführen [3].

In engster Zusammenarbeit mit offiziell bezeichneten Vertretern der Facharztgesellschaften wurden die Inhalte für die vier zweitägigen Kurse definiert. Anschliessend gelang es, sowohl hochkarätige juristische wie medizinische Referenten aus den universitären Fakultäten zu gewinnen, wie auch sehr praxiserfahrene Versicherungsjuristen und -mediziner. Selbst renommierte Patientenanwälte waren bereit, als Dozenten mitzuarbeiten. Damit konnte der Auftrag der FMH, die Ausbildungskurse unter Einbezug aller am Gutachterwesen Beteiligten neutral durchzuführen, voll erfüllt werden. Inzwischen geniessen die 4 modularen Gutachterkurse bei allen Institutionen, die sich mit Gutachten auseinanderzusetzen haben, grosse Anerkennung. Die Themen der einzelnen Module sind in den Tabellen 1-4 aufgeführt. Die Referentenlisten finden sich auf der Homepage der SIM (www.swiss-insurancemedicine.ch $\rightarrow$ Gutachterausbildung). Die routinemässig durchgeführten Kursevaluationen sind überwiegend gut bis sehr gut ausgefallen.
Seit August 2006 bis Dezember 2007 haben über 800 Teilnehmer die Module 1-3 besucht, 111 davon auf Französisch. 2008 werden wir insgesamt 10 Kurse durchführen, 2 auf Französisch. Die Ausbildung schliesst mit einem Zertifikat ab. Bedingungen sind: FMH-Facharzttitel, Absolvierung aller 4 Module, Eingabe eines selbsterstellten Gutachtens oder eines Berichtes von gutachterlichem Wert zur Beurteilung durch zwei Experten, einen medizinischen des gleichen Faches sowie einen juristischen. Am 4. Juli 2008 werden die ersten 100 Teilnehmer ihr Zertifikat erhalten, weitere $100 \mathrm{im}$ September. Auch die zukünftigen Kurse stehen unter dem Patronat der FMH und werden in deren Auftrag durchgeführt. Ende 2010 werden mehr als 800 Ärzte über das Zertifikat als medizinischer Gutachter verfügen.

\section{Die vier Ausbildungsmodule}

\section{Modul 1: Grundlagen zur Begutachtung}

- Der Gesundheitsschaden aus juristischer Sicht

- Kurzübersichten über UV, MV, IV, KV, BV

- Kurzübersicht Privatversicherung, Haftpflicht

- Arbeitsunfähigkeit, Erwerbsunfähigkeit, Invalidität, Verweistätigkeit und Zumutbarkeit, bezogen auf die verschiedenen Versicherungen (aus juristischer Sicht)

- Kausalität, Integritätsschaden, Genugtuung, bezogen auf die verschiedenen Versicherungen, sowie die Gliedertabelle (aus juristischer Sicht)

- Bemerkungen aus Sicht des Mediziners

- Gutachtermarkt und statistische Hilfsmittel

- Rechtliche Aspekte des Gutachtens/Gutachters

- Anforderungen an Gutachter und Ethik des Gutachters

- Aufbau eines Gutachtens

- Wertigkeit klinischer Befunde und die Sicherung der Diagnose

- 4 kasuistische Workshops

\section{Modul 2: Psyche und Soma}

in der Begutachtung

- Die Spannungsfelder des Gutachters

- Gesprächsführung durch den Gutachter

- Der Umgang mit Exploranden, Versicherern, Auftraggebern, Patientenanwälten 
- Häufige Verständigungsprobleme zwischen Medizinern und Juristen, oder wie tickt der Mediziner, wie der Jurist?

- Was ist eine psychische Krankheit: zur Bedeutung nosologischer Modelle für die Begutachtung

- Krankheitsbilder/Diagnosen im Grenzgebiet Psyche/Soma

- Verdeutlichung, Aggravation, Simulation, Versicherungsmissbrauch

- Posttraumatische Belastungsstörung und Anpassungsstörung

- Physiologie und Psychologie des Schmerzes

- Rückenschmerz und Psyche

- Juristische Aspekte zum Grenzgebiet Psyche/ Soma

- Unterschiedliche Fragestellungen im Grenzgebiet Psyche/Soma bezüglich der verschiedenen Versicherungsarten

- Umgang mit den sozialen Faktoren in bezug auf die verschiedenen Versicherungen sowie Aspekte der beruflichen Eingliederung

- Die Gesprächsführung des Gutachters, der Umgang mit Exploranden, Administration, Anwälten, Gerichten

- Umgang mit Verdeutlichung, Aggravation, Simulation und Versicherungsbetrug

\section{Modul 3: Fachspezifisches Modul}

- Chronische Schmerzzustände (Fibromyalgie, somatoforme Schmerzstörung)

- Vertebrale und radikuläre Syndrome: weiche und harte Facts aus rheumatologischer Sicht
- Verebrale und radikuläre Syndrome: weiche und harte Facts aus neurologischer Sicht

- Das kraniozervikale Beschleunigungstrauma

- Polytrauma, inkl. Schädelhirntrauma, St.n. Hypovolämie, Schock, Hypoxie

- Stellenwert psychologischer Testuntersuchungen, Möglichkeiten und Grenzen der Neuropsychologie

- Stellenwert biomechanischer Beurteilung und unfallanalytischer Gutachten

- 18 fachspezifische kasuistische Workshops

Modul 4: Fachspezifisches Schlussmodul

- Fatigue-Syndrom, Mobbingfolgen, Burn-out aus psychiatrischer Sicht

- Fatigue und Schläfrigkeit aus organischer Sicht

- Die (noch) nicht erklärbare Gesundheitsstörung, offene Diagnose im Rahmen der Begutachtung

- Stellenwert poly- und interdisziplinärer Gutachten

- Das kontradiktorische Gutachten

- Haftpflichtgutachten bei Kunstfehlern aus medizinischer und juristischer Sicht

- Der Kunstfehler aus Sicht des Rechtsdienstes der FMH

- Die Sicht des Patientenanwaltes und seine Wünsche an Gutachter (allgemein)

- Die häufigsten Fehler in Gutachten aus medizinischer, versicherungsrechtlicher und juristischer Sicht (mit Kasuistiken)

- 12 fachspezifische kasuistische Workshops 